\title{
Energy Conversion Mechanism and Parametric Analysis of Free Piston Engine Generator
}

\author{
Yanxiao $\mathrm{Li}^{1+}$, Jun Yang ${ }^{1}$, Zhengxing Zuo ${ }^{2}$ and Yongjian $\mathrm{Hu}^{1}$ \\ ${ }^{1}$ School of Automotive Engineering, Shandong Jiaotong University , Jinan 250357 , China \\ ${ }^{2}$ School of Mechanical Engineering, Beijing Institute of Technology, Beijing 100081, China
}

\begin{abstract}
The simulation model for free piston engine generator was set up in Matlab/Simulink and the working process simulation was conducted. According to the dynamic characteristic of the system, the energy conversion process was simplified in different stages of one stroke. On that basis, the effects of design parameters on the energy conversion process and operating state were analyzed.
\end{abstract}

Keywords: free piston engine, linear generator, energy conversion, parametric analysis.

\section{Introduction}

With the current energy and environmental pollution issues become more and more serious, as a novel internal combustion generator power system, the free piston engine for linear generator (FPEG) is attracting attention due to its advantages of compact design, multi-fuel feasibility, high power density, and short energy transfer path, etc [1]-[3]. It is a simpler and more efficient energy conversion device compared to the crank engine (CE), and it has become one of the preferred solutions for hybrid electric vehicles [4].

FPEG as a power generator has less load fluctuation, so it can be considered to work continuously under a single working condition [5]. Due to the removal of the crank-link mechanism and the inertial flywheel device, there is a strong coupling between the parameters of FPEG, and the changes of the design parameters would directly affect the system energy conversion process, thus affecting the piston movement and the operating state. In order to make the compression ratio and operating frequency meet the engine design requirements, it is necessary to deeply study the energy conversion mechanism and explore the relationship between operating state and design parameters. In this paper, a numerical model of the FPEG was developed in Matlab/Simulink and the dynamic characteristic was obtained. The energy conversion process of one stroke was divided into three stages and simplified in individual stages. The effect of design parameters on the energy conversion process and operating state were analyzed. This study provides a theoretical basis for the matching design and parameter optimization of FPEG prototype.

\section{Configuration and principle}

The configuration of a dual-piston FPEG considered in this paper is illustrated in Fig. 1. The prototype is comprised of two opposing internal combustion cylinders, each with its combustion chamber, scavenging ports, spark electrode and piston. A linear electric machine is integrated in the middle of two internal combustion cylinders. The two opposed pistons are connected to the mover of the linear electric machine, this component is the only moving part of the system. In the steady working state, the two-stroke engines on both sides ignite alternately, and the electrical current is generated from the alternator coils through the continuous back and forth movement of the mover. As the Fig. 1 shows, the piston motion is not restricted

\footnotetext{
+ Corresponding author: Tel.: +86 18806415108.
}

E-mail address: liyanxiao@163.com. 
by the motion of a rotating crankshaft, as known from crank engines, but that the piston can oscillate freely between the two double ended cylinders. It leads to a special trajectory of the FPEG piston movement.

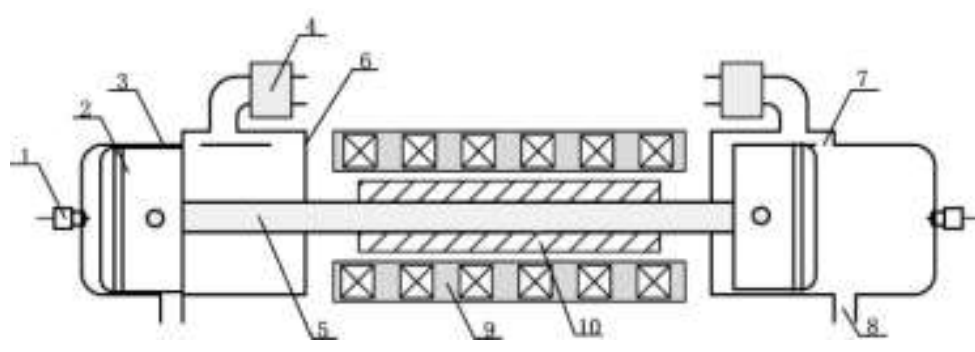

1. Spark plug; 2. Piston; 3. Cylinder; 4. Fuel supply system; 5. Connecting rod; 6. Scavenging case; 7. Scavenging port; 8 . Exhaust port; 9. Stator; 10. Mover.

Fig. 1: Configuration of FPEG

\section{Simulation modeling and methodology}

For the FPEG, the motion of the piston is not mechanically constrained but emerged as a result of realtime balance of the gas force in cylinder, electric load force and inertia force [6]. The FPEG does not employ any crankshaft mechanism and the purely linear motion leads to a very low side force on the piston, so the friction is ignored. By applying Newton's second law, gives:

$$
m \frac{d^{2} x}{d t^{2}}=\left(p_{\mathrm{L}}-p_{\mathrm{R}}\right) A-F_{\mathrm{e}}
$$

Where $m$ is the mass of moving component; $x$ is its displacement; $F_{\mathrm{e}}$ is the electric load force, $F_{\mathrm{e}}=K v, K$ is the electromagnetic damper coefficient of the linear generator and $v$ is the velocity of moving component; $A$ is the top area of the piston, and $A=\pi D^{2} / 4, D$ is the cylinder bore diameter; $p_{\mathrm{L}}$ and $p_{\mathrm{R}}$ are the gas pressure for each cylinder respectively. When the intake and exhaust ports are both closed, neglecting crevice flow and leakage, the first law of thermodynamics applied to the cylinder content becomes [5]:

$$
\frac{d p}{d t}=\frac{\gamma-1}{V} \frac{d Q}{d t}-\gamma \frac{p}{V} \frac{d V}{d t}
$$

Where $V$ is the volume of the cylinder; $\gamma$ is ratio of heat capacities; $Q$ is the total heat input.

In order to investigate the dynamic characteristic of the FPEG, A numerical model was developed in Matlab/Simulink, as shown in Fig. 2, and the equations were solved using Runge-Kutta solver with a fixed step size of $10^{-5}$. Before running the program, the main parameters of the FPEG prototype and the initial values are first entered into the program. The values used are listed in Table 1.

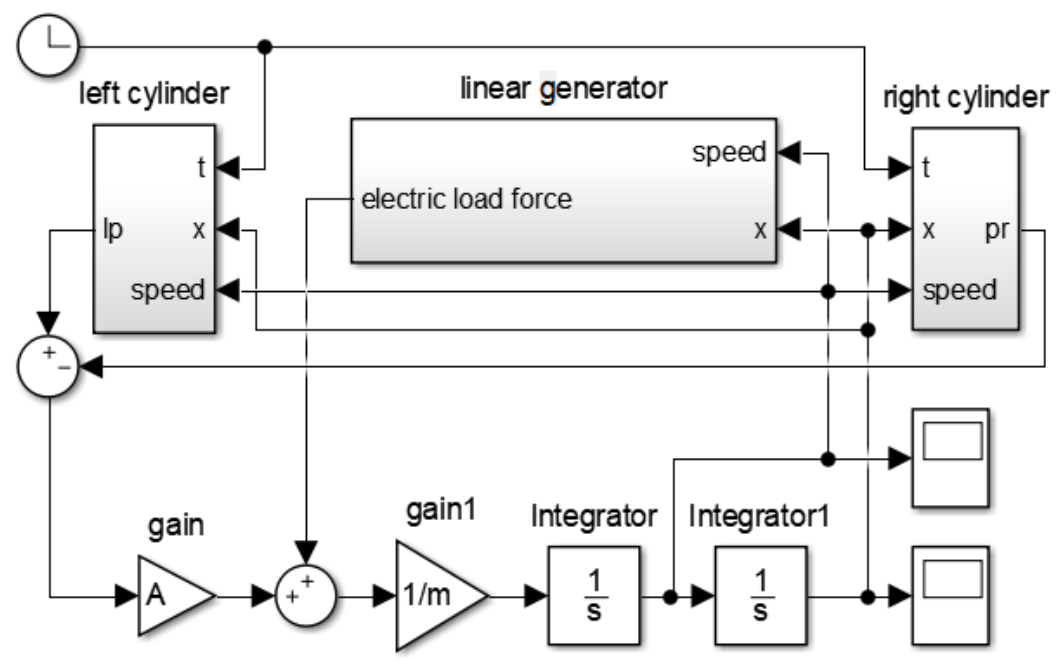

(a) The whole simulation model 

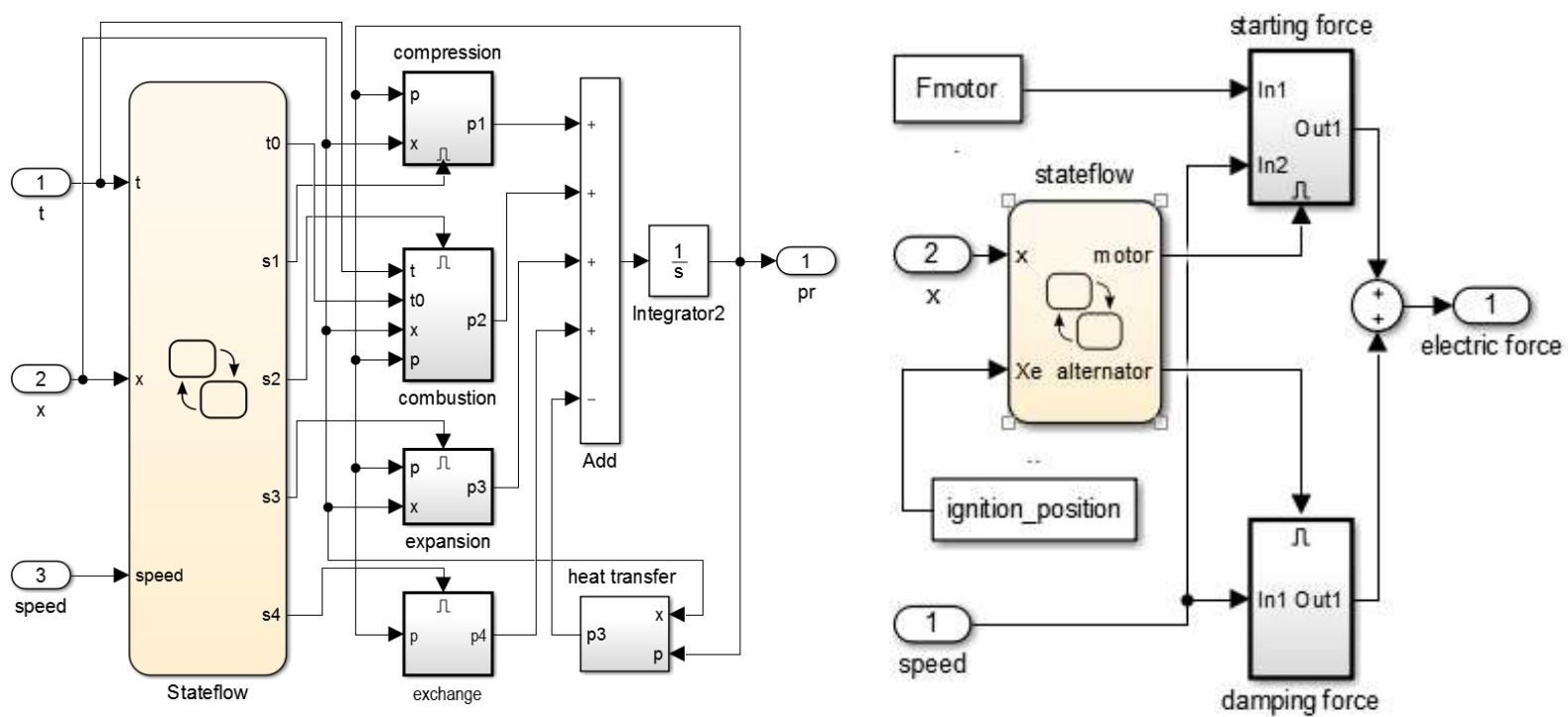

(b) The models of engine and electric machine

Fig. 2: Simulation model diagram of FPEG system

Table 1: Parameters of the FPEG

\begin{tabular}{lll}
\hline Parameters & Value & Unit \\
\hline Bore & 52.5 & $\mathrm{~mm}$ \\
Stroke & 68 & $\mathrm{~mm}$ \\
Effective stroke length & 34 & $\mathrm{~mm}$ \\
Moving mass & 5 & $\mathrm{~kg}$ \\
Intake air pressure & 1.2 & $\mathrm{bar}$ \\
\hline
\end{tabular}

Fig. 3 shows the piston speed loop over one engine cycle for the FPEG compared with a crank engine. The piston speed loop of the crank engine is determined by the crank system, so the piston motion is axisymmetric with zero velocity as the axis. For the FPEG, however, the piston speed is different at the same position before and after the dead centers. As shown in Fig. 3, the FPEG piston speed moving away from the dead center is much higher than that approaching the dead center, and the shape of piston speed loop is similar to a slanted round-rectangle with nearly piecewise linear characteristic.

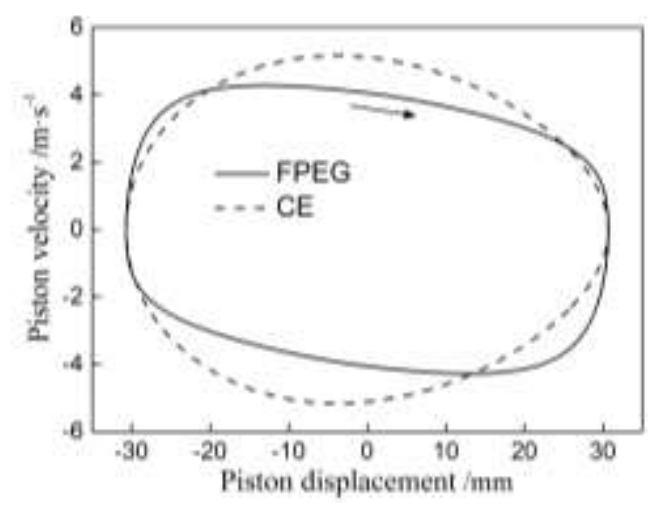

Fig. 3: Piston velocity vs displacement

\section{Energy conversion process analysis}

For convenience, one stroke of FPEG from left to right was selected as the research object. As shown in Fig. 4, the gas thrust generated by the fuel combustion in the left cylinder does positive work, which means that the thermal energy is inputted into the system and converted into the kinetic energy. The electric load force generated by the linear electric machine does negative work, which means that the kinetic energy of the 
moving component is converted into the electrical energy output. The gas thrust due to the volume change of left cylinder does positive work, which means that the compress energy is converted into the kinetic energy. The right cylinder gas thrust does negative work, which means that the kinetic energy is converted into the compress energy. The carrier of energy conversion between different forms is the kinetic energy of the moving component, which determines the movement of the FPEG piston.

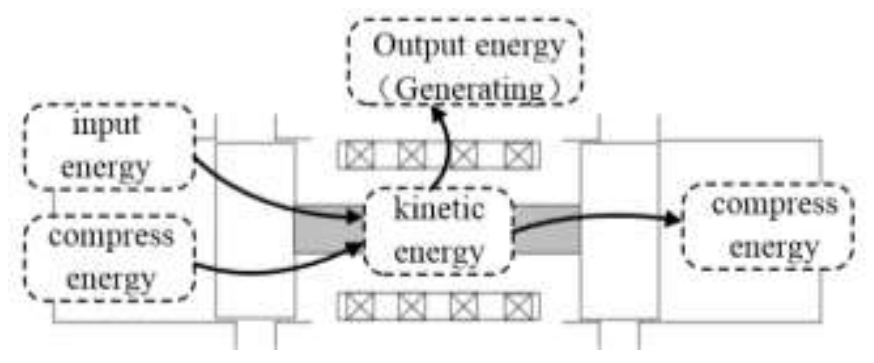

Fig. 4: Diagram of energy conversion process

Due to the FPEG is a complex nonlinear system, the energy conversion process of one stroke is divided into three stages according to the piecewise linear characteristic of piston speed loop (Fig. 3).

In the first stage, the heat energy from fuel combustion is rapidly released into the system and converted into the kinetic energy of the motion component. At the same time, most of the compress energy of the left cylinder is also converted to the kinetic energy. As the piston speed near the dead center is very low, the electric load force is small and the energy output of the system can be ignored. Obviously, the energy conversion form in this stage is that the most of the input energy and the compress energy are converted into kinetic energy. At the end of this stage the piston speed reaches its peak:

$$
E_{i}+E_{c} \approx \frac{1}{2} m v_{\text {max }}^{2}
$$

Where $E_{i}$ is the input energy to system; $E_{c}$ is the left cylinder compress energy; $v_{\max }$ is the peak velocity.

In the second stage, the position of the motion component is far from the two dead centers, therefore the changes of the compress energy of the two cylinders are fairly minor. Obviously, the energy conversion form in this stage is that the kinetic energy of the motion component is converted into electrical energy by the linear generator, gives:

$$
\int K v d x=\Delta \frac{1}{2} m v^{2}=\frac{1}{2} m\left(v_{1}+v_{2}\right)\left(v_{1}-v_{2}\right)
$$

Where $v_{1}, v_{2}$ are piston speed at both ends of $d x$; the following equation can be derived from Equation(4):

$$
\frac{d v}{d x}=-\frac{K}{m}
$$

Equation (5) indicates that the velocity decreases linearly in the second stage. The slope is proportional to the electromagnetic damper coefficient and inversely proportional to the moving component mass.

In the third stage, the motion component is getting closer to the right dead center and the residual kinetic energy is converted into the compress energy of the right cylinder.

\section{Effect of design parameters on energy conversion process and operating state}

The calculations of FPEG piston speed curves with different motion component mass are illustrated in Fig.5. According to Equation (3), lower moving component mass leads to higher peak speed at the end of the first stage. For the electromagnetic damper coefficient is constant, the higher speed level leads to the larger electric load force. Therefore, more energy output through the linear generator in the second stage and less residual kinetic energy in the third stage, which results in less compress energy at right dead center. According to the analysis above, higher moving mass leads to lower operating frequency and higher compression ratio, as shown in Fig. 6. 


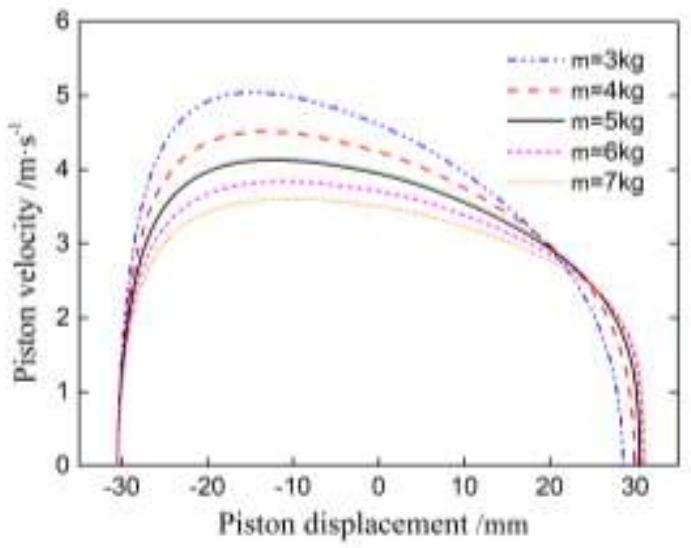

Fig. 5: Piston velocity with different

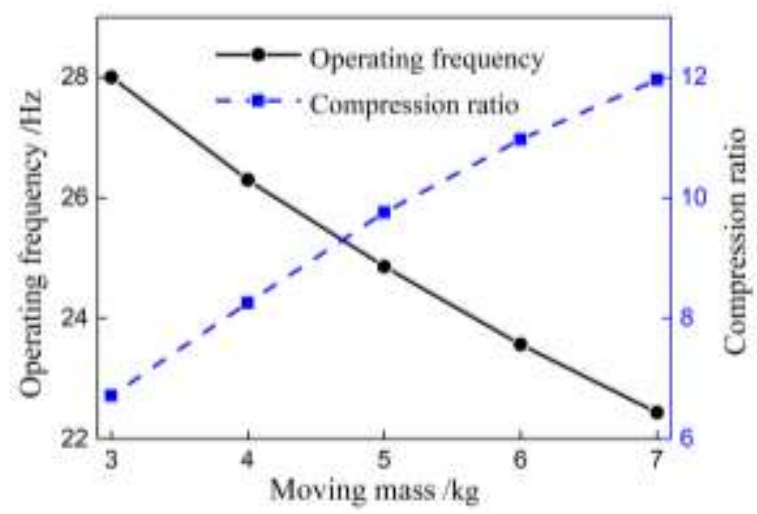

Fig. 6: Operating frequency and compression ratio motion component masses with different motion component masses

The calculations of FPEG piston speed curves with different cylinder bore are illustrated in Fig.7. A larger cylinder bore leads to more air intake and fuel injection, which means that the input energy to the system increases. According to equation (3), more input energy leads to higher peak speed at the end of the first stage. According to equation (5), under the condition that the motion component mass and the electromagnetic damper coefficient are constant, the curves corresponding to different cylinder bores have the same slope in the second stage. In the third stage, more residual kinetic energy leads to higher compress energy at right dead center. According to the analysis above, larger cylinder bore leads to higher operating frequency and higher compression ratio, as shown in Fig.8.

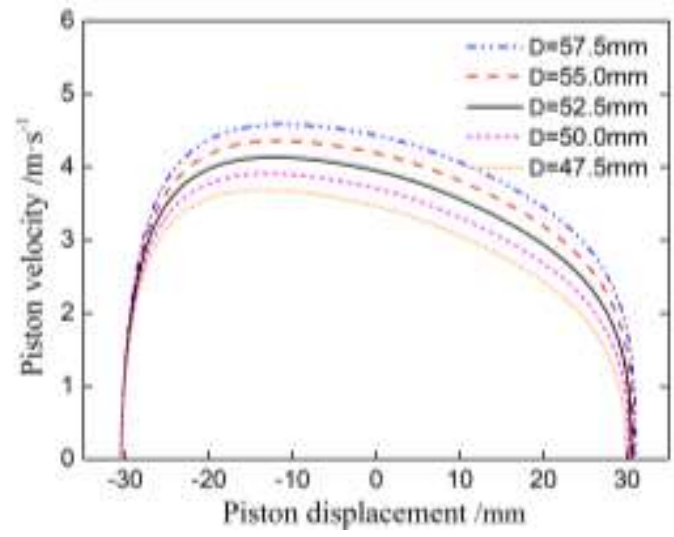

Fig. 7: Piston velocity with different 


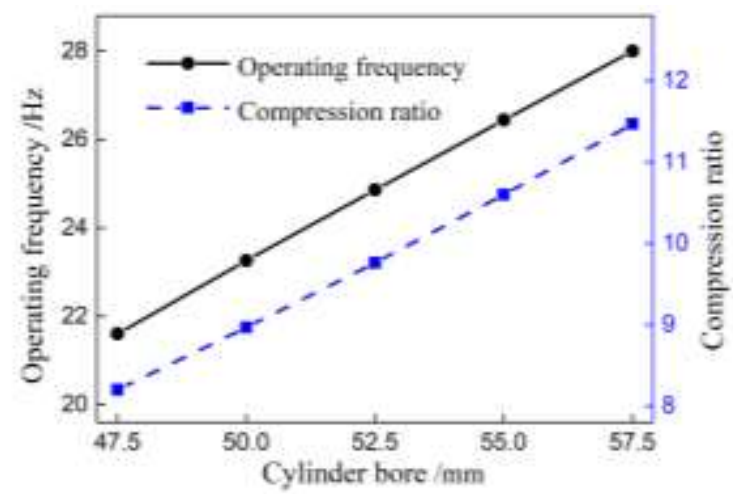

Fig. 8: Operating frequency and compression ratio cylinder bore with different cylinder bore

The calculations of FPEG piston speed curves with different electromagnetic damper coefficient are illustrated in Fig.9. As the change of the electromagnetic damper coefficient has no effect on the input energy of the system, the speed peaks of different curves are similar. In the second stage, larger electromagnetic damper coefficient leads to faster the motion component speed drops, which results in less residual kinetic energy in the third stage and less compress energy at right dead center. According to the analysis above, larger electromagnetic damper coefficient leads to lower operating frequency and lower compression ratio, as shown in Fig.10.

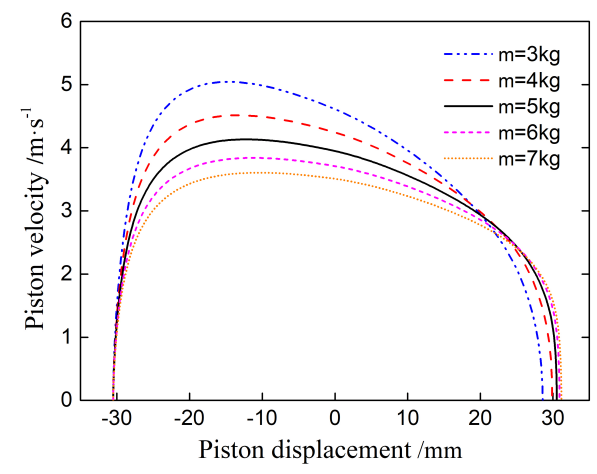

Fig. 9: Piston velocity with different

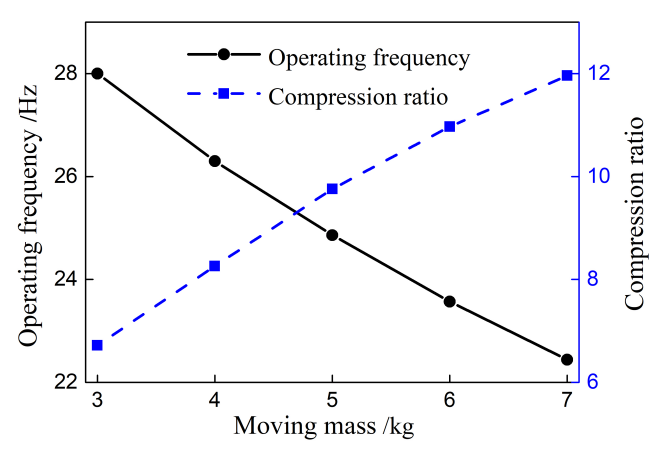

Fig. 10: Operating frequency and compression ratio electromagnetic damper coefficient with different electromagnetic damper coefficient

\section{Conclusions}

In this research, FPEG working process simulation was conducted and the piecewise linear characteristic of piston speed loop was found in the simulation results. On that basis, the energy conversion process of one stroke was analysed and the effect of design parameters on the operating state was obtained. It is indicated that: higher moving mass leads to lower operating frequency and higher compression ratio; 
larger cylinder bore leads to higher operating frequency and higher compression ratio; larger electromagnetic damper coefficient leads to lower operating frequency and lower compression ratio.

\section{Acknowledgements}

This project is supported by National Nature Science Foundation of China (No.61803231 and No. 51675043); Doctoral Scientific Research Initial Foundation and Nature Science Foundation of Shandong Jiaotong University (No. Z201633). We would like to thank the sponsors.

\section{References}

[1] B.H. Nguyen, L. Ocktaeck. A review of free-piston linear engines. Applied Energy, 2016, 178: 78-97.

[2] Y.X. Li, Z.X. Zuo, H.H. Feng, et al. Parameters matching requirements for diesel free piston linear alternator startup. Advances in Mechanical Engineering, 2015, 7(3): 1-8.

[3] Y. Woo, Y.J. Lee. Free piston engine generator: Technology review and an experimental evaluation with hydrogen fuel. International Journal of Automotive Technology, 2014, 15(2):229-235.

[4] M. R. Hanipah, R. Mikalsen, A. P. Roskilly. Recent commercial free-piston engine developments for automotive applications. Applied Thermal Engineering, 2015, 75: 493-503.

[5] J.L. Mao, Z.X. Zuo, W. Li, et al. Multi-dimensional scavenging analysis of a free-piston linear alternator based on numerical simulation. Applied Energy, 2011, 88(4): 1140-1152.

[6] Y.X. Li, Z.X. Zuo, H.H. Feng. Structural heat-transfer characteristics of free piston engine. Transactions of CSICE, 2018,36(1): 90-95. 